\title{
Generation of compressed air by cascaded thermocompressors - project status
}

\author{
Fabian Fischer, and Hans-Detlev Kühl*
}

TU Dortmund University, Chair of Thermodynamics, Emil-Figge-Str. 70, 44227 Dortmund, Germany

\begin{abstract}
Compressed air is one of the most widely used industrial energy sources, and its production by mechanical compressors is extremely expensive and, in addition, inefficient. Therefore, the utilization of waste heat, which accrues on a large scale in many industrial applications, by means of a thermocompressor is an obvious alternative. This contribution presents a design approach to experimentally investigate a reciprocating thermocompressor with a self-actuated overdriven free displacer. These experiments shall be the basis for the design and realization of a cascade of identical stages, which has so far only been investigated by means of analytical modelling and theoretical similarity considerations. The main advantage of this approach is its extreme constructive simplicity and the expected self-regulating effect, which both facilitate a cost-effective production and operation. As the minimization of dead volume and fluid friction losses is essential, the heat exchangers and the regenerator are plainly realized by an annular gap around the displacer in the proposed design, thus further increasing its simplicity. The design parameters are determined by an optimization based on an analytical model extended by various losses and heat transfer limitations.
\end{abstract}

\section{Introduction}

Compressed air is an important industrial energy source, but its mechanical generation is costly and consumes large amounts of primary energy [1,2]. At the same time, most industrial processes generate waste heat $[3,4]$, which is mostly discarded or used as low-grade heat for space heating only. One option to utilize such waste heat more efficiently is by reciprocating thermocompressors using air as the working fluid, which correspond to the displacer-heat exchanger system of a $\beta$ - or $\gamma$-Stirling engine, but feature two check valves instead of the compression piston.

This concept dates back to a 1935 US patent by V. Bush [5] and has so far been mainly considered for low-pressure applications such as artificial hearts [6-9], since the pressure ratio of a single-stage thermocompressor is severely limited by the temperature ratio and by dead volume effects. To support or substitute the generation of compressed air by conventional compressors, pressure levels of common industrial compressed air systems ranging from 6 to 10 bar must be reached. Therefore, a cascaded arrangement of several

\footnotetext{
*Corresponding author: hans-detlev.kuehl@tu-dortmund.de
} 
stages is required [10-12]. In the past, there have been various approaches to analytically describe, simulate, construct and test such machines, which have been reviewed by Fischer and Kühl [12]. The project currently in progress at the Chair of Thermodynamics of TU Dortmund University aims at investigating the possibility of generating compressed air from waste heat by means of a cascade of identical thermocompressor stages.

\section{Analytical cycle analysis}

To explain the general operating principle of a thermocompressor, Fig. 1 shows its schematic layout. The aforementioned similarity to the displacer-heat exchanger system of a $\beta$ - or $\gamma$ Stirling engine is visualized in detail here, as a displacer piston (1) divides a cylinder into a hot (2) and a cold (3) working space. The air, which acts as both the working fluid of the cycle and the product stream, is displaced between these working spaces via a duct consisting of a heater (7), a regenerator (8) and a cooler (9). According to the isothermal model, hot working space and heater are assumed to be kept isothermal at the high temperature $T_{h}$ and, correspondingly, cold working space and cooler at a low temperature $T_{c}$. Unlike the Stirling cycle, the cycle of the thermocompressor is an open cycle, since it conveys mass and the system gas mass changes periodically. Therefore, check valves $(5,6)$ are needed, which are located in the cold working space, as well as the displacer rod (4) [12].

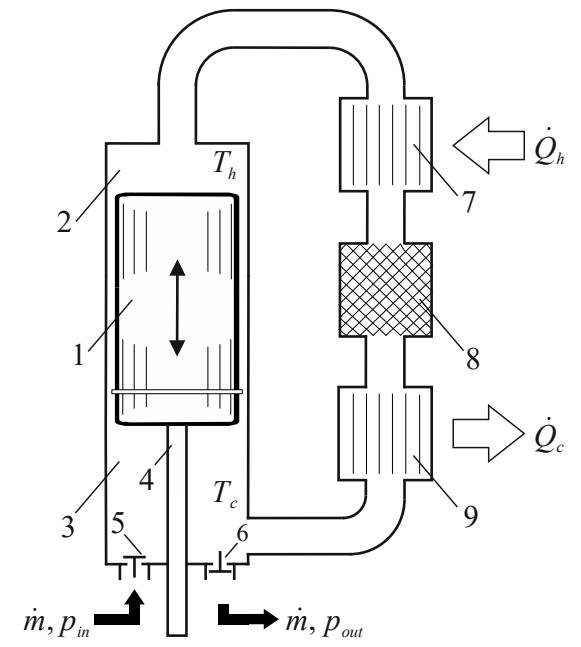

Fig. 1. Layout of a thermocompressor: (1) displacer, (2) hot working space, (3) cold working space, (4) displacer rod, (5) inlet valve, (6) outlet valve, (7) heater, (8) regenerator, (9) cooler [12].

Although it can also be characterized by a sequence of four states, the resulting ideal cycle differs significantly from the closed Stirling cycle, since the process steps in between these states are different. This is illustrated by the $\mathrm{p}, \mathrm{V}$-diagram shown in Fig. 2. Two of these states correspond to the upper (state 1) and the lower (state 3 ) end of stroke. The states in between are determined by the outlet pressure $p_{1}$ and the inlet pressure $p_{0}$. They correspond to the opening times of the outlet (state 2 ) and the inlet (state 4 ) valve. As a result, the cycle consists of two process steps with constant mass and variable pressure, and of two steps with constant pressure and variable mass. The following analysis of these changes of state is independent of the way how the displacer is actuated, which will be discussed in more detail in section 3 . Throughout process 1-2, the piston moves from the upper end of stroke downwards. As both valves are closed, the system gas mass is constant and the pressure rises, because gas is displaced from the cold to the hot working space, taking up heat in the regenerator [12]. 
In state 2, the system pressure reaches the outlet pressure, so that the outlet valve opens. Instead of a further pressure increase, mass is discharged, so that the system gas mass decreases and the ideal process 2-3 is isobaric [12].

When reaching the lower end of stroke in state 3, the displacer reverses its direction of motion. Both valves are closed again, and the system gas mass remains at its minimum value during process $3-4$. While the gas is displaced from the hot to the cold working space, it is cooled down in the regenerator. Therefore, the pressure decreases until the inlet pressure is reached in state 4 [12].

Here, the inlet valve opens, so that the subsequent process 4-1 is isobaric again. Instead of a further pressure reduction due to the ongoing displacement of gas from the hot to the cold working space, new gas enters the system via the inlet valve, and the system gas mass increases until it reaches its maximum in state 1 . At this point, the cycle is completed [12].

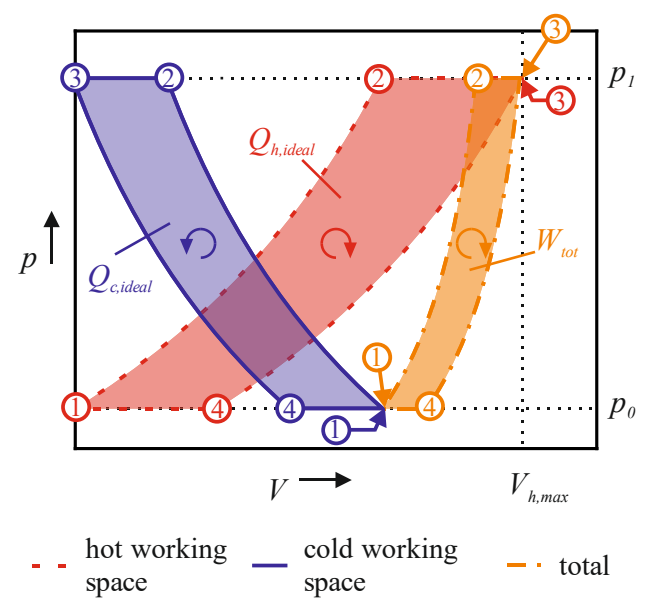

Fig. 2. p,V-diagram for an ideal thermocompressor process including process states and curves for the two working spaces and the total volume [12].

Several authors [13-20] developed analytical models to describe the thermodynamic cycle based on different simplifications. Fischer and Kühl [12] presented an analytical model including an arbitrary number of dead volumes with different temperatures and the effect of the displacer rod, which plunges into the cold working space and causes a change of the total cycle volume. For the development of this model, a generalized version of the well-known Schmidt analysis [21] was used. It implies the assumption of isothermal volumes, with a logarithmic mean temperature assumed for the perfect regenerator. The working fluid (air) is assumed to be an ideal gas. Furthermore, flow losses within the machine as well as in the valves are neglected, and steady-state operation is assumed [12].

On that basis, a function for the conveyed mass per cycle as a function of the pressure ratio $\Pi=p_{1} / p_{0}$ is determined:

$$
\Delta m=p_{0}\left(\Pi_{\max }-\Pi\right) \frac{B-D}{R}
$$

Here, $\Pi_{\max }$ is the maximum pressure ratio achievable for the given design and operating conditions in the limiting case when states 2 and 3 as well as 4 and 1 coincide and therefore, the valves do not open any more. $R$ denotes the specific gas constant.

$$
B=\frac{\widehat{V}}{T_{c}}(1-\rho+\theta+\sigma)
$$

can be interpreted as the mean sum of the temperature-related volumes and 


$$
D=\frac{\widehat{V}}{T_{c}}(1-\rho-\theta)
$$

as its amplitude. $\widehat{V}$ is the volume amplitude of the hot working space,

$$
\rho=\frac{A_{r}}{A_{D}}
$$

the ratio of the cross-sectional areas of the displacer rod and the displacer,

$$
\theta=\frac{T_{c}}{T_{h}}
$$

the ratio of the cold and the hot working space temperatures, $T_{c}$ and $T_{h}$, and

$$
\sigma=\frac{T_{c}}{\widehat{V}} \sum \frac{V_{d, i}}{T_{d, i}}
$$

the dimensionless sum of the temperature-related dead volumes. In the latter, $V_{d, i}$ represents any dead volume $i$ within the machine, and $T_{d, i}$ the corresponding temperature. As $B$ and $D$ characterize the machine (or rather the underlying ideal cycle), the maximum pressure ratio

$$
\Pi_{\max }=\frac{B+D}{B-D}
$$

can be calculated on their basis [12].

Fischer and Kühl [12] present a detailed derivation of the equation

$$
Q_{h, \text { ideal }}=2 \widehat{V} p_{0} \frac{\left(\Pi_{\max }-\Pi\right)}{\Pi_{\max }-1} \ln \Pi
$$

for the absorbed heat per cycle $Q_{h, \text { ideal }}$, which includes the influence of the dead volume and the displacer rod. It corresponds to the $\mathrm{p}, \mathrm{V}$ loop area for the hot working space in Fig. 2. Fig. 3 shows a plot of eq. (8) versus $\Pi$ for an exemplary thermocompressor specification. A maximum is clearly evident, the existence of which is proven in [12]. This maximum is the optimum operating point of such a machine, as the exergy of the compressed air stream directly depends on the absorbed heat, if an ideal reversible cycle is assumed. The optimum pressure ratio $\Pi_{o p t}$ can be approximated by $\sqrt{\Pi_{\max }}$.

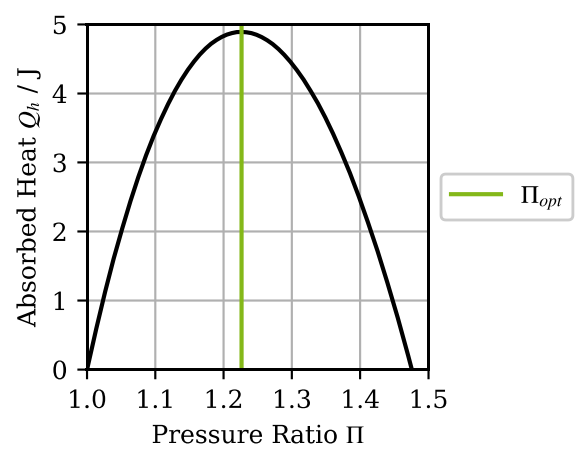

Fig. 3. Absorbed Heat $Q_{h}$ per cycle for an exemplary thermocompressor design as a function of the pressure ratio $\Pi\left(T_{c}=293 \mathrm{~K}, \rho=0.15, \sigma=0.4, \theta=0.51, \hat{V}=1000 \mathrm{~cm}^{3}\right)$ [12]. 


\section{Concept for a cascade of identical stages}

The value of $\Pi_{\max }$ is theoretically limited by the inverse of the temperature ratio $\theta$ even for an ideal cycle without any dead volumes, and since these are indispensable for a sufficient heat transfer at acceptable flow losses, it is even further reduced. The same applies to the pressure ratio $\Pi_{\text {opt }}$ at the optimum operating point. Therefore, among others $[5,10,11]$ Fischer and Kühl [12] suggest to resort to a cascade of multiple thermocompressor stages.

The innovative idea within their approach is a combination of identical stages in order to make the design as simple, flexible and, above all, cost-effective as possible. Since the power density of a thermocompressor is low due to the limitations of $\Pi_{\max }$ and $\Pi_{o p t}$, it is essential here to ensure that all stages operate close to $\Pi_{\text {opt }}$. In this case, the mass conveyed per cycle is a linear function of their respective inlet pressures $p_{0}$ according to eq. (1), and therefore, their operating frequencies must be adjusted inversely to the these due to the constraint of an identical mass flow in all stages. Similarity considerations reveal that several characteristic dimensionless quantities, such as Reynolds-, Valensi-, Mach- and Stanton Numbers, are thus actually maintained constant. Therefore, identical heat transfer conditions are found in the heat exchangers and the regenerators, although pressure and density vary by roughly one order of magnitude from the first to the last stage [12].

Unfortunately, this is not true for frictional pressure drop effects, the relative impact of which decreases from the first to the last stage. However, it is believed that this similarity violation may be largely compensated by reducing the heater temperature from the first to the last stage. This profitably matches a decreasing thermal load with an increasing mechanical stress and simultaneously allows an extraction of heat at a gliding temperature from the enthalpy flow of a hot stream (e. g. a flue gas flow) as indicated in Fig. 4 [12].

A further design simplification may be achieved by using overdriven free displacers. However, unlike those in overdriven free piston or Ringbom Stirling engines, these should undergo elastic bounces at the ends of stroke, thus preserving their kinetic energy. They are expected to automatically adapt their frequencies, since the generated work $W_{\text {tot }}$ that actuates them (visualized by the $\mathrm{p}, \mathrm{V}$ loop area for the total cycle volume in Fig. 2) equals $\rho \times Q_{h \text {,ideal }}$ and therefore also decreases according to eq. (8) as soon as $\Pi_{o p t}$ is exceeded. This self-control mechanism is being investigated for multiple stages in current simulation studies.

Because of the decoupled operating frequencies, this concept requires additional buffer volumes between the stages, which may additionally be used to cool down the air so that the coolers in the stages might be downsized. Such a layout is shown in Fig. 4. The stepped design of the displacer rod simplifies the balancing of forces, because this way, the overall force is the same as if a mean pressure $\bar{p}$ would act on the total cross section of the rod [12].

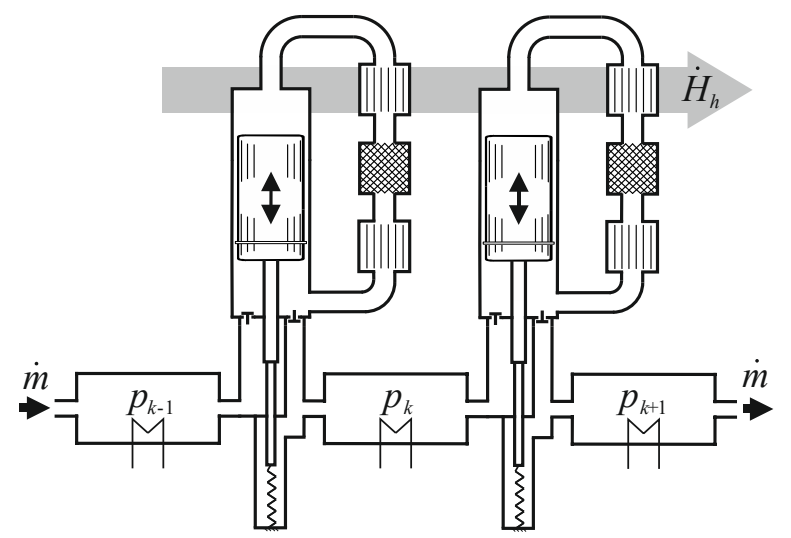

Fig. 4. Layout of a cascade of free-displacer thermocompressors including cooled buffer volumes [12] 


\section{Analytical approach for the equation of motion of the overdriven free displacer}

Fischer and Kühl [12] developed an analytical model to describe the motion of the overdriven free displacer in a thermocompressor. It is based on the balance of forces,

$$
m_{D} \ddot{x}+j \dot{x}+A_{R} p(x)+C_{G} x=A_{R} \bar{p}
$$

Here, $m_{D}$ is the mass of the displacer and $p(x)$ the pressure as a function of the displacer position . Friction forces are assumed to be linearly speed-dependent and are quantified by the constant factor $j$. This assumption is required to maintain linearity and thus analytical solvability of eq. (9). Assuming that the entire generated work is dissipated by this force, it is possible to estimate the operating frequency on the basis of $j$ (or vice versa). Introduction of an additional spring constant $C_{G}$ was found to be advantageous to partially compensate the force exerted by the cycle pressure on the cross section $A_{R}$ of the rod and thus minimize phases with a decelerating overall force along the stroke path. When the valves are closed, the cycle acts on the rod like a gas spring with a negative spring constant, the so-called cycle spring constant. Since it is pressure-dependent, the spring constant $C_{G}$ should preferably also be realized by a gas spring, so that the compensation effect is the same in all stages [12].

As the pressure function $p(x)$ differs due to the varying system gas mass and the opening of the valves, the equation of motion has to be solved on four sections corresponding to the processes presented in chapter 2. During the closed processes 1-2 and 3-4, the pressure $p(x)$ is dependent on the displacer position. During the open processes 2-3 and 3-4, $p(x)$ is constant and equal to the outlet or inlet pressure. The analytical solution of the resulting system of equations is possible only if the following simplifications are applied [12]:

- $\quad$ Linearized pressure functions

- $\quad$ Linearly speed-dependent friction force (i.e., $j=$ const.)

Furthermore, the following assumptions were made to facilitate the mathematical treatment and to visualize the major parameter effects in the resulting solutions more clearly:

- Operation close to $\Pi_{o p t}$, so that valves open close to mid-stroke position

- Conveyed mass is small compared to system gas mass, so that system gas mass may be approximated by a mean value in some instances

- Negative cycle spring constant is the same during both closed processes

- Gas spring constant $C_{G}$ is half the absolute value of the cycle spring constant

Fig. 5 shows exemplary curves for the dimensionless piston position $\xi=x / \hat{X}$, related to the stroke amplitude, as a function of time for different displacer masses. As the damping constant $\delta=j t_{0} / 2 m_{D}$ is dependent on a time constant $t_{0}$, the friction coefficient $j$, and the the displacer mass $m_{D}$, it varies accordingly. Evidently, the velocity fluctuations decrease with increasing displacer mass. It can be shown that the same applies for an increasing stroke amplitude. This is a preferable effect, since the cycle time is significantly shortened or the frequency is increased, respectively, and the locus curve of the piston position approaches a triangle function for its way upwards and downwards. This has a positive effect on the friction losses, which are dependent of the square of the velocity even in case of the above-mentioned simplification concerning the friction coefficient, so that less work is required to operate the system at a given frequency. In general, it can be stated that fluid friction has an extreme impact on the performance of such a machine. This is impressively highlighted by the fact that, considering the analytical solution, halving the friction coefficient results in more than a doubling of the exergy flow and the operating frequency [12]. 


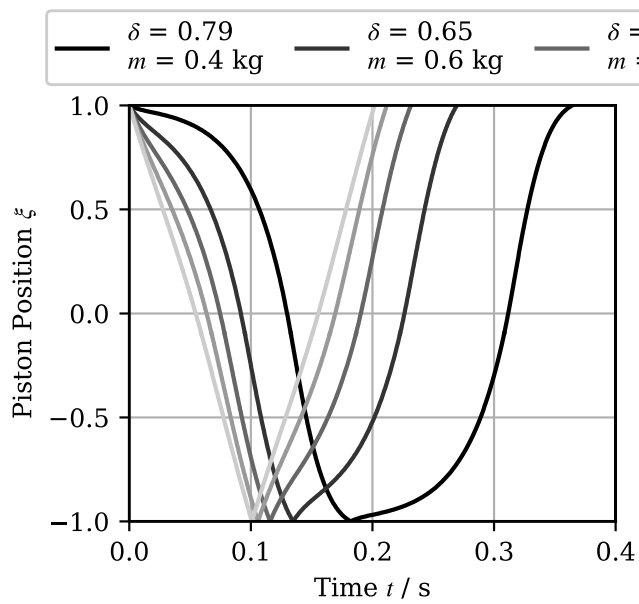

$\begin{array}{lll}\delta=0.53 & & \delta=0.41 \\
m=0.9 \mathrm{~kg}\end{array} \quad-\begin{aligned} & \delta=0.25 \\
& m=1.5 \mathrm{~kg}\end{aligned}$\begin{tabular}{l}
$m=4.0 \mathrm{~kg}$ \\
\hline
\end{tabular}

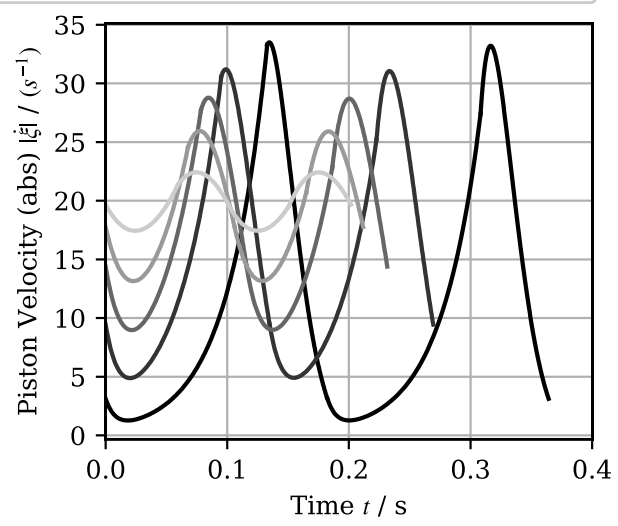

Fig. 5. Dimensionless displacer position $\xi$ and absolute velocity $d \xi / d t$ for different displacer masses $m_{D}$ as a function of time $t[12]$.

\section{Construction concept for a laboratory-scale machine}

At present, a machine is being built at the Chair of Thermodynamics of TU Dortmund University to experimentally investigate the fundamental operating principle of the concept described in section 3. Initially, only one single stage will be realized, primarily to validate the theory regarding the different operating points and to study the motion of the displacer and the stability of the operating points. Furthermore, the analytical model as well as numerical simulations of a single stage are to be compared to the experimental results.

To take a decision between a radially shifted and a concentric arrangement, a crucial design aspect must be discussed. Because both dead volume and fluid friction losses have an extreme impact on the machine performance according to the considerations in chapter 2 and 4 , a concentric arrangement was chosen, so that flow passages are minimized. For the same reason, Walker [22] suggests to replace complex types of heat exchangers and regenerators by an annular gap in case of small-scale machines operating below $500 \mathrm{rpm}$ and at relatively low pressure levels. This makes the general design simple, apart from the problem of machining sufficiently thin-walled components to reduce axial heat conduction losses.

The design resulting from this proposal and the previously described concept is shown in Fig. 6. Please note that Fig. 6 is not a true-to-scale technical drawing, but merely a schematic illustration. This particularly applies to the wall thickness of the displacer and cylinder wall as well as the annular gap, which are actually around $1 \mathrm{~mm}$ and would therefore not be clearly visible. Furthermore, all measurement equipment such as e.g. pressure and displacement sensors and the related mountings are omitted for simplicity reasons.

The machine can be divided into two sections, the active cycle volume and the buffer spaces. The former consists of the cyclically varying cylinder volumes and the annular gap, which is the only dead volume apart from small, shallow counterbores ensuring that the thin metallic valve reeds are not hit by the displacer. To maximize the number of parallel check valves and thus minimize their flow losses, they are distributed on both the cylinder base and the displacer base. The inlet buffer space is located directly below the cold cylinder, so that the inlet air flow can enter the working space through the inlet valves in the cylinder base. The outlet flow is directed through the outlet valves in the displacer base and thus enters the interior of the displacer, which is already part of the outlet buffer space due to the connection via the hollow rod. The lower section of this space also accommodates the various springs. 


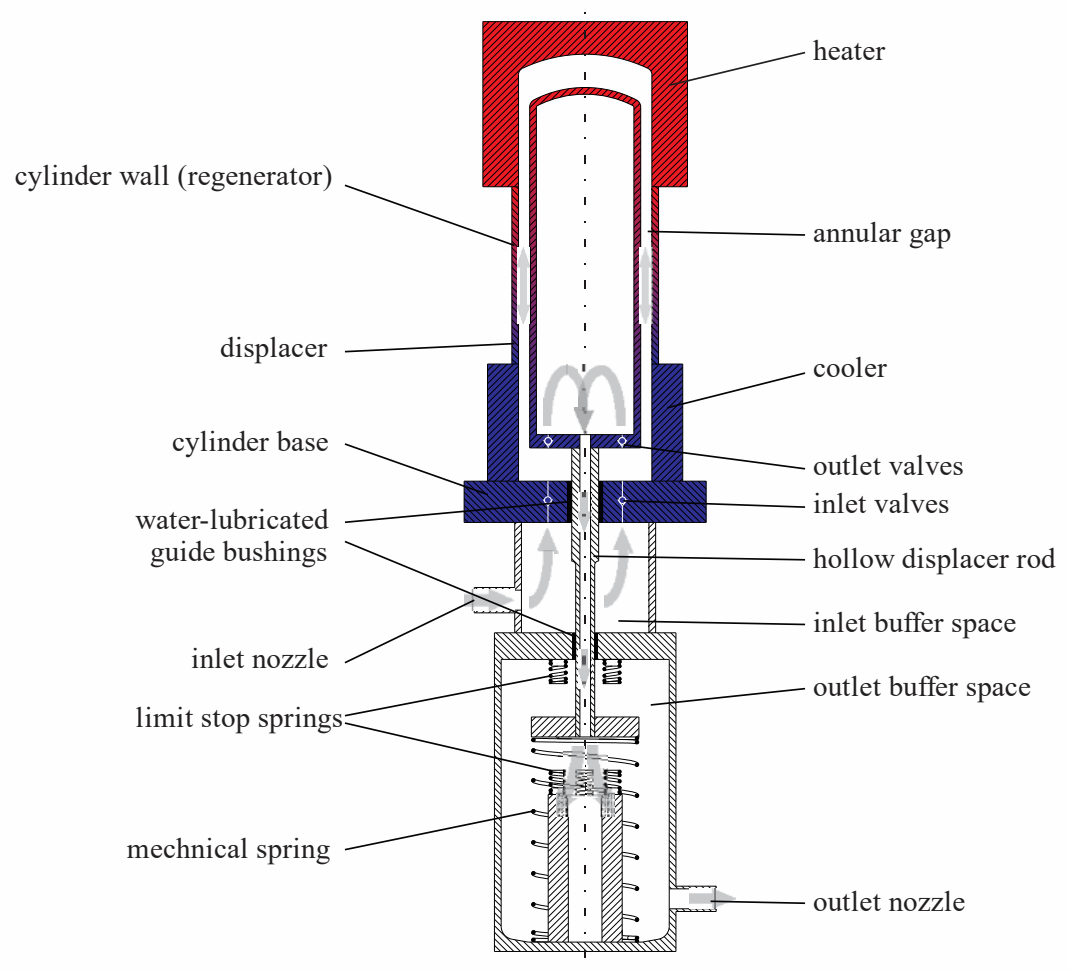

Fig. 6 Simplified schematically drawing of the laboratory-scale machine including the path of the airflow. The width of the annular gap is not to scale for reasons of better visualization.

The elastic limit stops are realized by an arrangement of sufficiently stiff springs placed in the outlet buffer space. A disk which is mounted at end of the displacer rod hits these springs at the upper and the lower end of stroke. The mounting of the springs is designed to allow for a precise adjustment of the limit stops.

For the first experiments, the gas spring mentioned above is replaced by a conventional spring with a corresponding spring constant for reasons of simplicity. Additionally, this gives the opportunity to investigate the influence of the spring constant by simply exchanging the spring. The spring is also used to maintain the displacer in the center position at standstill.

Due to the compression along the cascade, the dew point temperature of the moisture content in the air will gradually increase. In contrast to conventional adiabatic compressors, this compression is almost isothermal because of the repeated intermediate cooling via the coolers inside the stages and subsequently via the aforementioned additional cooling in the outlet buffer spaces, which helps to reduce the size and the dead volume of the former. It may be easily realized by e. g. inserting a finned tube coil into the buffer space, since no dead volume constraints apply. The compressed air will presumably reach its lowest temperature here, and so, condensation is expected to occur in the outlet buffer spaces of the higher stages. The condensate accumulating at the bottom shall be used to provide water-lubrication to the seal and guide bushings at the displacer rod, which are made of a low-friction polymer also ensuring good dry-running properties. The water may be fed to the bushings e.g. via capillaries, since the hydrostatic pressure difference is far lower than that between these bushings and the outlet buffer space. The water is expected to finally accumulate in the inlet buffer space of the stage, as the pressure is lowest here. Any excess water not needed for the bushings may be directly fed to this space via a simple float valve control. So, any condensate accumulating in the highest stage will eventually leave this stage via the inlet nozzle and flow 
into the outlet buffer space of the previous stage due to gravity effects. In a cascade, the condensate formed in the higher stages is therefore expected to follow the pressure gradient in counterflow to the conveyed air, thus providing lubrication to all stages as required, even if there is no condensation in first ones, and to finally exit via the inlet nozzle of the first stage. For the first experiments with a single stage, water will have to be supplied to the outlet pressure buffer space externally to simulate the condensate formation in the higher stages.

The dimensioning of the machine is generally based on the isothermal model outlined in section 2 and described more extensively in [12]. For this purpose, the model was expanded to consider heat transfer effects within the machine, conduction via the displacer and cylinder wall and flow pressure losses in the gap, so that an iterative solution was required. The final parameters were determined by an optimization of the exergy flow of the compressed air at the optimum operating point. Previously, the displacer diameter was set to $82.5 \mathrm{~mm}$ and the stroke volume to $0.267 \ell$, based on Walker's suggestions concerning the sizing of machines with annular gaps [22], the goal of building a relatively small machine for the first tests and the fact that the resulting stroke of $50 \mathrm{~mm}$ allows the desired displacer movement according to the analytical calculations. Gap width, operating frequency, the lengths of the cooler, regenerator and the heater and the rod diameter were optimization parameters. It is found that the flow in the gap is laminar for the optimum, which obviously does not provide the best heat transfer conditions, but underlines the sensitivity of the design to pressure losses. As already suggested [12], increased thermal losses are thus accepted rather than flow losses.

The aluminum-made cooler will be connected to tap water, so that the low temperature will be approximately $15-20^{\circ} \mathrm{C}$. The cylinder and the displacer wall forming the regenerative annulus are made as thin as possible to minimize heat conduction losses, and stainless steel is chosen as material. The wall thickness of about $1 \mathrm{~mm}$ is not imposed by the mechanical strength, but rather by the technical limitations in machining thin-walled shells in single-part production. Seamless tubes with suitable inner diameter are used here as semi-finished products. After brazing flanges to the ends and thus increasing their stiffness, the outer diameter can be further reduced by turning. The aluminum-made heater is equipped with an electrical heating wire for easiest control. It is designed for a maximum heater temperature of $300{ }^{\circ} \mathrm{C}$. According to the calculations, the thermocompressor should absorb $60 \mathrm{~W}$ of heat at the optimum operating point and provide an output exergy flow of $2.35 \mathrm{~W}$ in return while running at a frequency of about $200 \mathrm{~min}^{-1}$ and generating a pressure ratio of 1.17 .

\section{Conclusion and outlook}

An experimental laboratory-scale thermocompressor with an overdriven free-displacer has been designed based on the concept suggested by Fischer and Kühl [12]. For the first experiments, a relatively simple design including an annular gap taking over the functions of the heat exchangers and the regenerator is considered to be best suited. After completion, the functionality of the machine, which so far could only be predicted on the basis of analytical calculations and simulations, will be investigated experimentally. The results of these first experiments will be used to continuously improve the construction and to confirm the results of the analytical considerations concerning performance and stability of operating points. In parallel, simulations are being carried out to prepare for the second phase of the project, which involves the extension of the experimental setup to a cascade of at least three stages. This will give the opportunity to analyze the self-regulation capabilities of the system.

This research was funded by the German Research Foundation (DFG), grant number KU 755/5-1. 


\section{References}

1. P. Radgen, E. Blaustein, Compressed air systems in the European Union (LOG_X Verlag, Stuttgart, 2001).

2. M. Unger, P. Radgen, Energy Efficiency in Compressed Air Systems: A review of energy efficiency potentials, technological development, energy policy actions and future importance, in: Bertoldi, Paolo, Proceedings of the 10th International Conference on Energy Efficiency in Motor Driven Systems (EEMODS' 2017), Rome, Italy, 207-233 (2018).

3. L. Miró, S. Brückner, L.F. Cabeza, Mapping and discussing Industrial Waste Heat (IWH) potentials for different countries, Renew. Sustain. Energy Rev. 51 (2015), 847-855 https://doi.org/10.1016/j.rser.2015.06.035.

4. M. Papapetrou, G. Kosmadakis, A. Cipollina, U. La Commare, G. Micale, Industrial waste heat: Estimation of the technically available resource in the EU per industrial sector, temperature level and country, Appl. Therm. Eng 138 (2018), 207-216 https://doi.org/10.1016/j.applthermaleng.2018.04.043.

5. V. Bush, Apparatus for Compressing Gases, US Patent 2,157,229 (1935).

6. W.R. Martini, The Thermocompressor and its Application to Artificial Heart Power, in: ACS, Proc. 4th IECEC, Washington, D.C., USA, 107-114 (1969).

7. B.M. Gibson, C.J. Hornbeck, R.C. Longworth, L.T. Harmison, Bypass gas actuated thermocompressor as an implantable artificial heart power source, in: SAE, Proc. 6th IECEC, Boston, MA, USA (1971).

8. J.C. Moise, M.I. Rudnicki, R.J. Faeser, Development of a thermocompressor power system for implantable artificial heart application, in: ASME, Proc. 8th IECEC, Philadelphia, PA, USA, 511-535 (1973).

9. M.A. White, Miniature Stirling Engines for Artificial Heart Power, in: AIChE, Proc. 18th IECEC, Orlando, FL, USA, 694-701 (1983).

10. A.A. Kornhauser, Analysis of an idealized Stirling thermocompressor, in: IEEE, Proc. 31st IECEC, Washington, DC, USA, 1331-1336 (1996) https://doi.org/10.1109/IECEC.1996.553909.

11. S. Thomas, E.J. Barth, Multi-Stage Modeling of a Stirling Thermocompressor, in: ASME, Proceedings of the ASME/BATH 2017 Symposium on Fluid Power and Motion Control (FPMC2017), Sarasota, FL, USA (2017) https://doi.org/10.1115/FPMC2017-4320.

12. F. Fischer, H.-D. Kühl, Analytical model for an overdriven free-displacer thermocompressor, Appl. Therm. Eng 185 (2021), 116251 https://doi.org/10.1016/j.applthermaleng.2020.116251.

13. G.Y. Umarov, V.S. Trukhov, I.A. Tursunbaev, Prospects for using dynamic thermocompression converter in solar power plants, Appl. Sol. Energy 10 (1974), 53-56.

14. A.G. Popov, V.S. Trukhov, Determination of the Thermodynamic Parameters of a Thermal Compressor, Appl. Sol. Energy 13 (1977), 9-14.

15. A.P.M. Glassford, Adiabatic cycle analysis for the valved thermal compressor, J. Energy 3 (1979), 306-314 https://doi.org/10.2514/3.48007.

16. P. Arquès, Thermodynamical cycle analysis of gas in a Thermocompressor, in: ANS, Proc. 33rd IECEC, Colorado Springs, CO, USA (1998).

17. P. Arquès, Piston movement in thermocompressor, in: AIChE, Proc. 32nd IECEC, Honolulu, HI, USA, 1003-1008 (1997) https://doi.org/10.1109/IECEC.1997.661906.

18. H. Karabulut, Thermodynamic Analysis Of Bush Engine, Gazi Univ. J. Sci. 16 (2003), 135-144. 
19. M.J. Edwards, R.B. Peterson, Modeling and Thermodynamic Cycle Performance of a Miniature Reciprocating Thermocompressor, in: AIAA, Proceedings 3rd IECEC, San Francisco, CA, USA (2005).

20. E. Blagin, A.I. Dovgyallo, D.A. Uglanov, S.S. Dostovalova, Thermodynamic calculation of a thermomechanical compressor, VESTNIK of the Samara State Aerospace University 12 (2014), 25-30 https://doi.org/10.18287/1998-6629-2013-03-2(41)-25-30.

21. H.-D. Kühl, Verallgemeinerte thermodynamische Beschreibung regenerativer Gaskreisprozesse, Dissertation, Universität Dortmund, Lehrstuhl für Thermodynamik (VDI-Verlag, Düsseldorf, 1990).

22. G. Walker, Stirling-cycle machines (Clarendon Press, Oxford, 1973). 